\title{
Molecular Probes in Tandem Electrospray Ionization Mass Spectrometry: Application to Tracing Chemical Changes of Specific Phospholipid Molecular Species
}

\author{
Hiroko Tominaga ${ }^{1}$, Tomoe Ishihara ${ }^{1}$, A. K. M. Azad Shah ${ }^{2}$, Rumiko Shimizu ${ }^{3}$, Arnold N. Onyango ${ }^{4}$, \\ Hideyuki Ito ${ }^{5}$, Toshinori Suzuki ${ }^{6}$, Yasuhiro Kondo ${ }^{1}$, Hiroshi Koaze $^{7}$, \\ Koretaro Takahashi ${ }^{2}$, Naomichi Baba ${ }^{*}$ \\ ${ }^{1}$ Division of Bioscience, Graduate School of Natural Science and Technology, Okayama University, Okayama, Japan \\ ${ }^{2}$ Sdivision of Marine Life Science, Graduate School of Fisheries Sciences, Hokkaido University, Hakodate, Japan \\ ${ }^{3}$ Faculty of Pharmaceutical Sciences, Kobe Gakuin University, Kobe, Japan \\ ${ }^{4}$ Jomo Kenyatta University of Agriculture and Technology, Nairobi, Kenya \\ ${ }^{5}$ Faculty of Health and Welfare Science, Okayama Prefectural University, Okayama, Japan \\ ${ }^{6}$ School of Pharmacy, Shujitsu Univerisity, Okayama, Japan \\ ${ }^{7}$ Department of Food Science, Obihiro University of Agriculture and Veterinary Medicine, Hokkaido, Japan \\ Email: "babanaom-10@t.okadai.jp
}

Received July 17, 2013; revised August 17, 2013; accepted September 15, 2013

Copyright (C) 2013 Hiroko Tominaga et al. This is an open access article distributed under the Creative Commons Attribution License, which permits unrestricted use, distribution, and reproduction in any medium, provided the original work is properly cited.

\begin{abstract}
New ionization and detection techniques in mass spectrometry have been successfully applied for efficient analyses of complex biological systems. It is, however, still difficult to trace structural changes of a specific molecular species in such systems. In the present study, a molecular probe strategy in combination with tandem electrospray ionization mass spectrometry has been examined using synthetic deuterium-labeled phosphatidylcholine hydroperoxide (PC-OOH/D3) and ethyl-labeled phosphatidylcholine having docosahexaenoic acid side chain (DHA-PC/Et). Administration of a mixture of PC-OOH/D3 and DHA-PC/Et to human blood and human skin surface, followed by extraction and analysis with collision-induced tandem electrospray ionization mass spectrometry demonstrated that metabolites of both molecular probes can be detected simultaneously with strict selectivity. The present method is also found to be useful in tracing chemical changes of the unstable docosahexaenoyl group on the surface of processed fish. The activity of phospholipase A2 can also be assessed using a phospholipid molecular probe with a linoleoyl and a deuteriomethyl group via selective detection of the lyso-phospholipid product by mass spectrometry. The advantage of the present method is that no chromatographic separation is required and analysis can be performed under strictly the same condition for different molecular probes, affording multiple data by one experiment. The present strategy may be useful for tracing time-dependent phenomena in dynamic phospholipid biochemistry, and can be widely used for any biological and food systems.
\end{abstract}

Keywords: Molecular Probe; Stable Isotope; Mass Spectrometry; Electrospray Ionization; Lipid Peroxidation; Lipid Oxidation; Skin Surface

\section{Introduction}

Many kinds of structurally-related phospholipid species 1 (Scheme 1) occur in biological systems due to numerous combinations of different fatty acids at $s n-1$ and $s n-2$ positions and the existence of different polar head groups $[1,2]$. Regioisomeric relations with different fatty acyl groups at $s n-1$ and $s n-2$ positions also increase the number of phospholipid species. The major fatty acids include palmitic, stearic, oleic, linoleic, linolenic, arachi-

*Corresponding author. donic, icosapentaenoic and docosahexaenoic acids, and the major polar head groups are choline phosphate, serine phosphate, ethanolamine phosphate, glycerol phosphate and inositol phosphate. The different phospholipid species usually have different roles in biological systems. They may localize different subcellular particles, cell membranes and tissues, or translocate according to changes in biological micro-environments. More complex is that phospholipid molecules change their structure moment by moment according to the changes in physiological conditions $[3,4]$. 


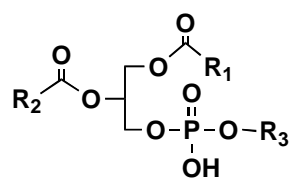

1

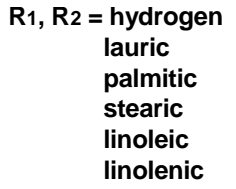

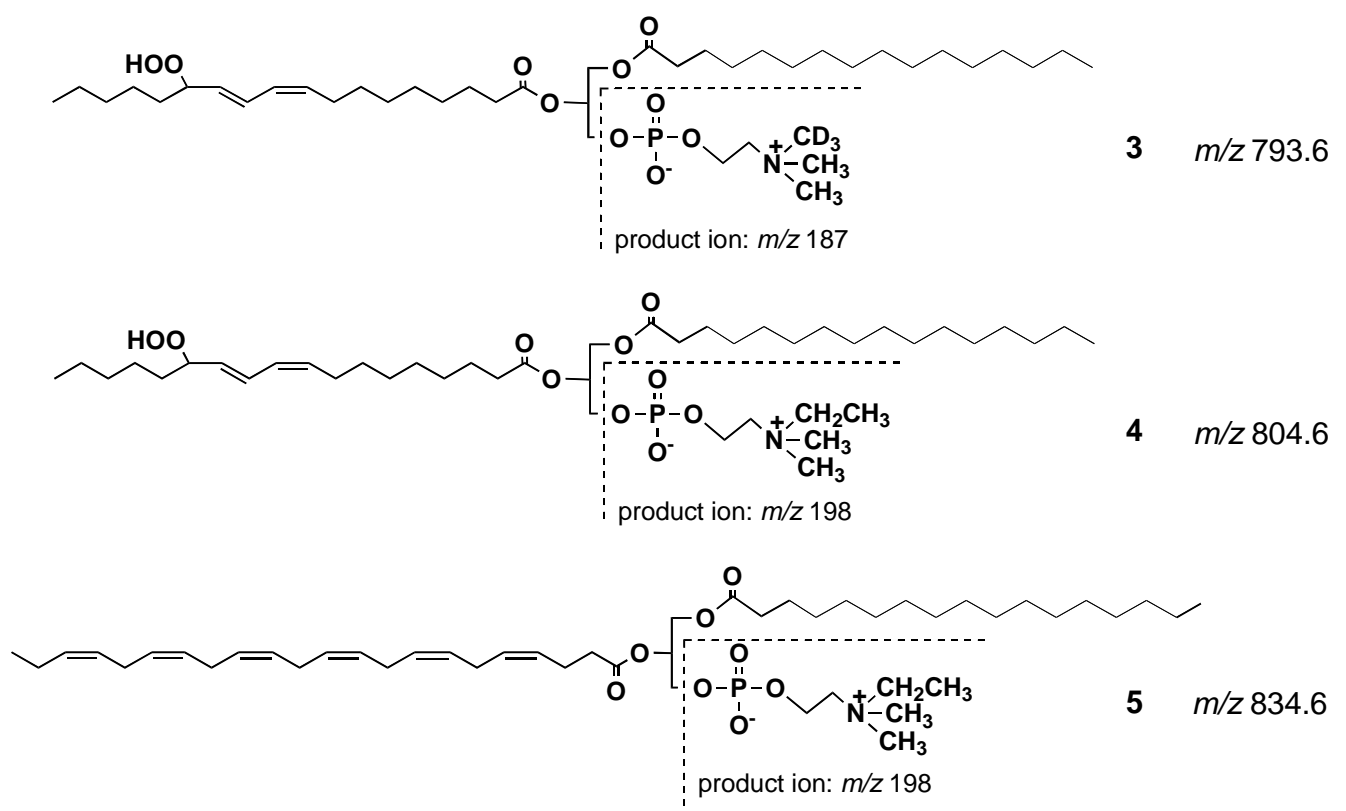

Scheme 1. Structure of phosphatidylcholine 1 (general structure) and 2 (natural phospholipid hydroperoxde), and synthetic phospholipids 3 - 5. Theoretical $m / z$ for 2 - 5, and observed $m / z$ of a product ion produced from 2 - 5 in collision induced ESI MS are given in this scheme.

A number of methods have been developed for the study of different aspects of phospholipids in biological systems. Fluorescence-labeled phospholipid molecular probes in combination with fluorescence microscopy have been instrumental in studies on the localization and translocation of specific phospholipids. For example, a phosphatidylcholine bearing a fluorescent chromophore was shown to be internalized from the cell surface into erythrocytes infected with malaria parasite [5]. The internalization of the fluorescent molecular probe was not due to endocytosis but a rapid trans-bilayer lipid flip-flop at the infected erythrocyte membranes, followed by monomer diffusion to the parasites. Electron paramagnetic resonance (EPR) of spin-labelled lipids has been demonstrated to be a versatile method for studying lipidprotein interactions in cell membranes. As reviewed by
Marsh [6], the use of spin-labeled phospholipids molecular probes has enabled determination of the stoichiometry of lipid-protein interactions, and selectivity of the proteins for different lipid species. Phospholipids labeled with radio-active atoms have also greatly contributed in classical but extremely sensitive lipid analysis in combination with chromatographic methods.

The rapid development of mass spectrometry has enabled speedy mapping of complex metabolites in a large scale using only a small amount of biological samples. The problems of complex biological phenomena such as apoptotic mechanism involving lipid peroxidation have been solved [7-11]. Based on the mass spectrometry technique, lipidomics studies have revealed close relations between biological lipid profiles and diseases $[12,13]$. Despite the development of such highly power- 
ful techniques, it is still almost impossible to trace chemical changes of a specific phospholipid species in the complex mixture of biological samples. In this review, we will briefly describe use of synthetic phospholipid molecular probes in combination with electrospray ionization mass spectrometry for studying oxidative changes of phospholipid species in complex biological systems including human blood, human skin and processed foods.

\section{Mass Spectrometry-Assisted Tracing of Chemical Changes in Phospholipid Molecular Probes}

The significance of lipid peroxidation in biological systems has been highlighted in recent years due to the unexpectedly broad biological activity of the peroxidation products, including both harmful and salutary effects $[14,15]$. Among the biological oxidized phospholipid species, PC-OOH such as $\mathbf{2}$ occur in mammalian blood at very low concentrations, and they are believed to be deeply involved in aging, physiological disorders and diseases [16-19] including Alzheimer's disease [20]. Most of the current knowledge on the chemistry and biochemistry of lipid oxidation has relied on simple in vitro models. Under such conditions, the hydroperoxides are very unstable due to the inherent sensitivity of the hydroperoxy group against radical initiators, and their degradation cascades are extremely complex. However, the process of formation of the phospholipid hydroperoxides in vivo as well as their metabolism and physiological significance are not fully understood due to the lack of highly sensitive analytical techniques. Although luminescence detectors can detect the phospholipid hydroperoxides at very low concentration, the products of hydroperoxide degradation are insensitive to this detector. Moreover, while mass spectrometry can detect these products, it usually requires time-consuming multifaceted pretreatments, and the separation of each degradation product in the biological samples. A possible solution to this problem is the use of a synthetic molecular probe for the specific PC-OOH molecule. Thus, we synthesized an unnatural phosphatidylcholine hydroperoxide $(\mathrm{PC}-\mathrm{OOH} / \mathrm{D}, 3)$ that has a deuteriomethyl group in place of one of three methyl groups at the nitrogen atom in the choline moiety. In this molecule, any chemical changes at the polyunsaturated fatty acyl chain may not be affected even if one of the three $N$-methyl groups in natural $\mathrm{PC}$ is replaced by a deuterium group. A strong advantage of this strategy is that even in the presence of complex biological phospholipid mixture, this unnatural phospholipid could be detected with strict selectivity in precursor ion scan mode of collision-induced tandem electrospray ionization mass spectrometry (ESI MS). Scheme 2 shows the principle of the precursor ion scan mode. The molecular probe $3(250 \mu \mathrm{g}, 320 \mathrm{~nm})$ was mixed with human blood $(0.5 \mathrm{ml})$ from a 22 year old healthy female volunteer, and incubated at $20^{\circ} \mathrm{C}$ for 5 min in nitrogen atmosphere. The initial concentration of 3 in the blood was $630 \mathrm{nM}$. After the incubation period, neutral lipids including triglycerides were extracted from the blood by washing with acetone supplemented with a trace of the anti-oxidant, butylated hydroxytoluene (BHT). BHT prevents further auto-oxidation of hydroperoxy polyunsaturated fatty acyl groups in $\mathbf{3}$ and those of natural phospholipids in blood during the washing. The total polar phospholipid in the residue was extracted three times with a mixture of $\mathrm{CHCl}_{3} / \mathrm{CH}_{3} \mathrm{OH}$ (1:2) containing a trace of BHT. After removing the solvent under reduced pressure, the residue was dissolved in a mixture of $\mathrm{CH}_{3} \mathrm{CN} / \mathrm{CH}_{3} \mathrm{OH} / \mathrm{H}_{2} \mathrm{O}(215: 194: 16)$ with $0.1 \%$ ammonium acetate and submitted to ESI MS analysis. When we scanned precursor ion scan mode at $\mathrm{m} / \mathrm{z} 187$, a signal for 3 was clearly observed at $\mathrm{m} / \mathrm{z} 793.8$ (Figure 1(A)), indicating that the deuterated phosphatidylcholine hydroperoxide 3 could be detected even in the presence of a complex mixture of natural lipids in the blood. In addition to the major signal at $m / z$ 793.8, a new signal appeared at $\mathrm{m} / \mathrm{z} 777.8$ that was less than 793.8 by 16 mass unit $(\mathrm{mu})$. Since the atomic mass of oxygen is 16 , the molecular species of the peak should have $\mathrm{C}-\mathrm{OH}$ instead of $\mathrm{C}-\mathrm{OOH}$. This deoxygenation is well known to occur in vitro in the homolytic cleavage of $\mathrm{OOH}$ group affording PC-OH/D3 6 (Scheme 3) [21]. A small signal is found at $\mathrm{m} / \mathrm{z} 775.8$ as a shoulder. This value is less than 793.8 by $18 \mathrm{mu}$ indicating that the molecular species is a carbonyl form 7 (Scheme 3) via dehydration of 3. We previously reported that $\mathrm{C}-\mathrm{OOH}$ was subjected to easy dehydration to afford carbonyl form $\mathrm{C}=\mathrm{O}$ such as 7 as a major product [22]. On the other hand, the formation of $\mathbf{6}$ was larger than 7 in the blood in the present study indicating that chemical change of the lipid hydroperoxides in simple model experiments may not fully reflect the fate of these products in complex biological systems. A new signal at $\mathrm{m} / \mathrm{z} 653.8$ (Figure 1(A)) that is less than 777.8 by $124 \mathrm{mu}$

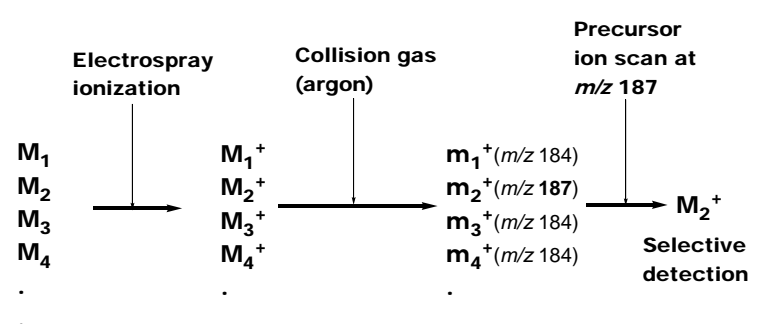

$\begin{array}{lll}\begin{array}{l}\text { A mixture of } \\ \text { different } \\ \text { molecules }\end{array} & \begin{array}{l}\text { Ionized } \\ \text { molecules }\end{array} & \begin{array}{l}\text { Flagment ions from } \\ \text { lonized molecules }\end{array}\end{array}$

Scheme 2. Selective detection of a specific molecular species by collision induced tandem electrospray mass spectrometry. 


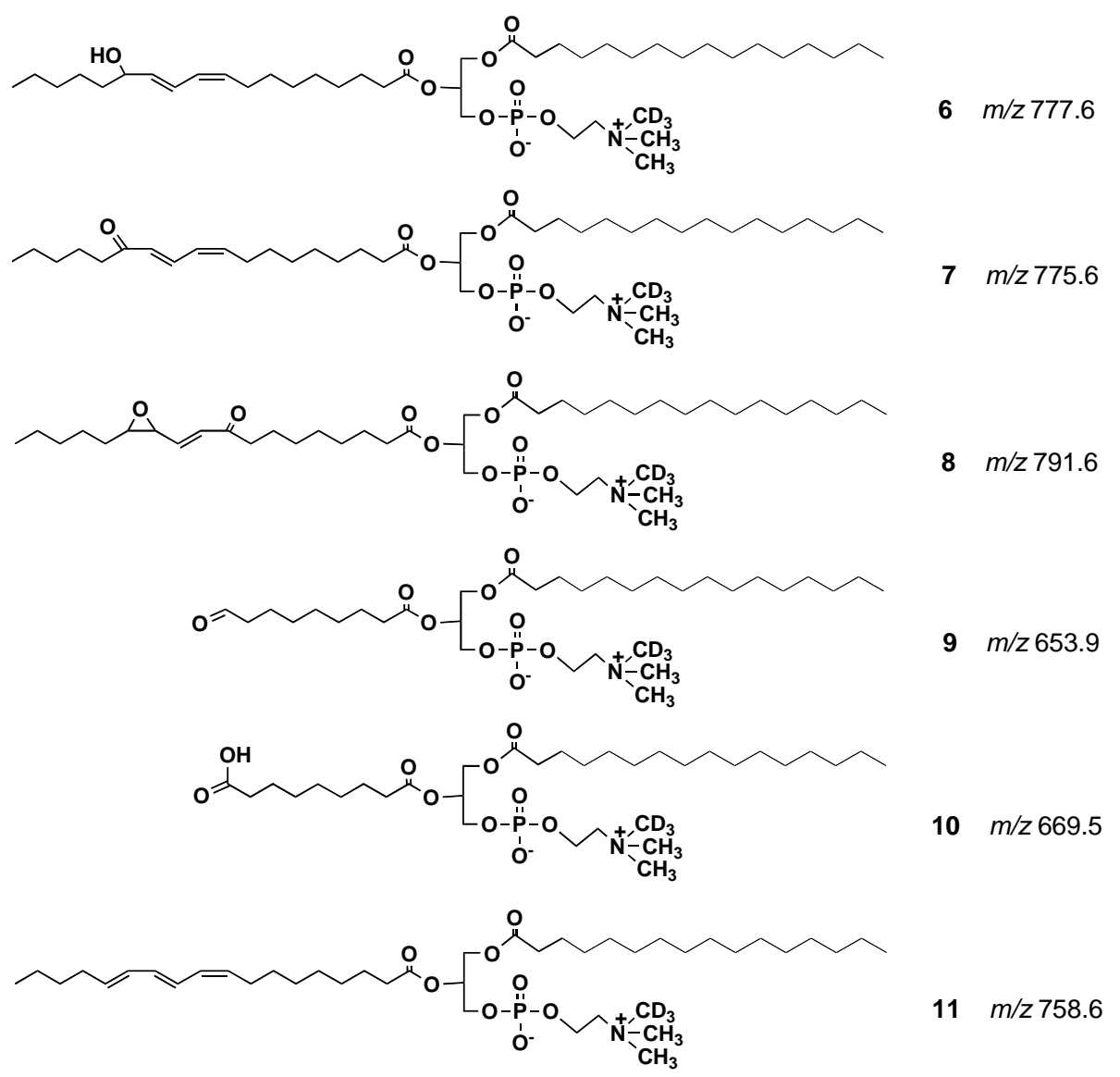

Scheme 3. Possible structure of degradation products formed from PCOOH/D3 3 . All the $\mathrm{m} / \mathrm{z}$ values were calculated from their protonated form.

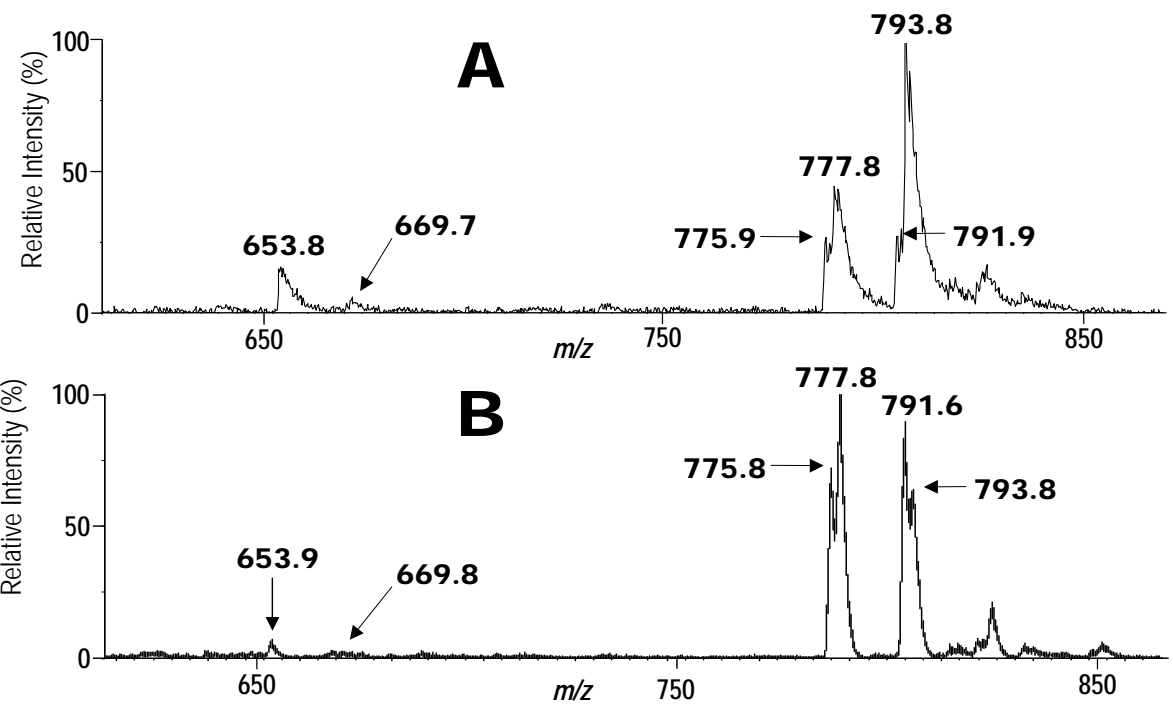

Figure 1. Two spectra for the phospholipid fraction recovered from the human blood obtained in precursor ion scan mode at $\mathrm{m} / \mathrm{z}$ 187. A) Blood of a healthy female volunteer (22 years old); B) Blood of a healthy male volunteer (66 years old). Signals of phospholipid bearing $\mathrm{CD}_{3}$ are seen in these spectra. A peak at $\mathrm{m} / \mathrm{z} 793.8$ is of unchanged intact phopholipid hydroperoxide 3 , and other signals are of degradation products formed from 3 in the blood.

was assigned as 2-(9-oxononanoyl)-deuterio-PC(PC-CHO) 9. The natural 2-(9-oxononanyl)-PC is known to be a major product of the decomposition of the corresponding linoleoyl-PC hydroperoxide 2, albeit the mechanism of 
this conversion has been unclear. The molecular species that gave a signal at $\mathrm{m} / \mathrm{z} 791.6$ was speculated as an oxo-epoxide 8 in Scheme 3 . This metabolite may be formed from 3 via the corresponding alkoxyl radical and a hydroperoxy-epoxide. Aldehydes such as $\mathbf{9}$ are known to undergo further non-enzymatic oxidation to their corresponding carboxylic acids $[21,23]$. Therefore, the molecular species that has $\mathrm{m} / \mathrm{z} 669.8$ was deduced to be an acid species $\mathrm{PC}-\mathrm{COOH} / \mathrm{D}_{3} 10$ (Scheme 3) since this molecular mass is bigger than that of aldehyde $\mathrm{PC}-\mathrm{CHO} / \mathrm{D}_{3}$ 9 by 16 mu. Oxidized PC that had a carboxyl group instead of the aldehyde group was also detected as a fluorescence derivative from human blood by Kern et al. [24]. The degradation profile of $\mathrm{PC}-\mathrm{OOH} / \mathrm{D} 33 \mathrm{in}$ blood from a 66 years old male volunteer is shown in spectrum $\mathbf{B}$ (Figure 1). It was similar to that of the blood from 22 years female volunteer spectrum A (Figure 1). More important is that this decomposition profile is close to that obtained by use of the molecular probe 3 , which has an ethyl group in place of the deuteriomethyl group in $\mathbf{4}$, confirming the reliability of this method [21]. Thus, if two molecular probes, M1 and M2 (such as 3 and 4), give different product ion mass-to-charge ratio $(\mathrm{m} / \mathrm{z})$, p1 and $\mathrm{p} 2$, respectively, the parent ion scan mode at $\mathrm{p} 1$ should give a signal of M1 at $m / z$ p1 with strict selectivity even in the presence of M2. In the same way, scanning at $\mathrm{m} / \mathrm{z}$ p2, we can detect a signal of M2 alone. When we apply a mixture of these two molecular probes M1 and M2 to a biological tissue, the sample can, after incubation, be analyzed by parent ion scan mode at $\mathrm{m} / \mathrm{z}$ pl or p2 affording dual spectral data obtained under strictly the same physiological condition. This new idea suggested that the molecular probe strategy might be useful for analysis of complex biological systems and might be expanded to multiple dimensional analytical systems in combination with ESI MS.

Docosahexaenoic acid (DHA) is a kind of lipid that is abundant in salt-water fish and has multi-functional properties for our health. An important chemical feature of DHA is that it has a non-conjugated all-cis-polyolefinic structure which makes it very unstable against oxidative stress. It undergoes rapid free radical-mediated oxidative degradation. Lipid oxidation products have long been considered to be undesirable against our health. Some of them, however, have been demonstrated in recent years to show rather salutary effects, such as activation of the immunological system [25]. Therefore, the chemistry and biochemistry of DHA oxidation are of great interest in recent years. However, the isolation and structural characterization of the peroxidation products formed in vivo from DHA-PC in particular appears to be almost impossible, and no study has been reported so far. Aiming at a solution of this problem, we synthesized DHA-PC/Et 5 (Scheme 1) as another molecular probe in which one of the methyl groups on the nitrogen atom was replaced by an ethyl group. This molecular probe provides a product ion at $\mathrm{m} / \mathrm{z} 198$ in tandem ESI MS instead of 184 for natural PC 1 or 2 or 187 for PC-OOH/D3 3. Therefore, as shown in Figure 2, upper, we considered that when a mixture of $\mathbf{3}$ and $\mathbf{5}$ was applied on a complex biological system, both the molecular probes are exposed to exactly the same environment being subjected to structural modifications in each. These modified PC species can be detected in a strictly selective manner in one ESI MAS measurement at $m / z 187$ for 3 and $m / z 198$ for 5 even in the presence of complex mixture of natural phospholipids. Thus, a mixture of $\mathbf{3}$ and $\mathbf{5}$ were applied on human blood from a 66 years old healthy volunteer as an example. After the incubation for $6 \mathrm{~h}$ under the same condition as for the first experiment, the lipid fraction was extracted from the blood with a mixture of $\mathrm{CHCl}_{3}$ / $\mathrm{CH}_{3} \mathrm{OH}(2: 1)$ containing a trace of BHT and the solution was submitted to precursor ion scan mode at $\mathrm{m} / \mathrm{z} 187$ for 3 or 198 for 5 in tandem ESI MS. As shown in spectrum $\mathrm{C}$ in Figure 2, a number of signals are observed between $\mathrm{m} / \mathrm{z} 450-600$ in addition to the major signals between $\mathrm{m} / \mathrm{z} 773$ - 798 when measurement was done at $\mathrm{m} / \mathrm{z} 187$. Although, structural assignment for all signals in the region of $\mathrm{m} / \mathrm{z} 450$ - 600 was not possible in terms of the oxidative fragmentation of hydroperoxy acyl group alone, if we assume that hydrolytic cleavage of the acyl group at $s n-1$ position of the glycerol backbone occurs affording a lyso-PC having smaller molecular mass, it is possible to assign some of the signals in the region of $\mathrm{m} / \mathrm{z} 450$ - 600 to possible structures (structures not shown). On the other hand, when measurement was done at $\mathrm{m} / \mathrm{z} 198$, a strong signal of intact DHA-PC/Et 5 (Scheme 1) appeared at $\mathrm{m} / \mathrm{z} 834.7$ as the only major peak (Figure 2, spectrum D). The small signal at $\mathrm{m} / \mathrm{z} 524.4$ in this spectrum could be assigned as protonated lyso PC 19, indicating that hydrolysis of the ester bond occurred to a small extent. This indicates that no significant degradation of DHA-PC/Et occurred at the unstable polyolefinic side chain in the phospholipid in the blood. However, it is not surprising since phosphatidylcholine bearing DHA is one of the major components of cell membranes.

Next, we focused on the fate of PC hydroperoxide 3 and DHA-PC/Et 5 at human arm skin surface. A mixture of 3 and 5 were applied on human skin surface of a 66 years healthy volunteer. After the exposure for $6 \mathrm{~h}$ at $20^{\circ} \mathrm{C}$ including sun light exposure for $1 \mathrm{~h}$, the lipid fraction was extracted with ethanol and the solution was submitted to precursor ion scan mode at $\mathrm{m} / \mathrm{z} 187$ for 3 or 198 for 5 in ESI MS. For the scan at $m / z$ 187, three major peaks were observed as seen in spectrum $\mathrm{E}$ in Figure 3. A signal at $m / z 793.8$ is for intact PC-OOH/D3 3. A signal at $\mathrm{m} / \mathrm{z} 777.6$ is for hydroxy PC 6 (Scheme 3) and 759.6 is consistent with product 11 (Scheme 3) which 


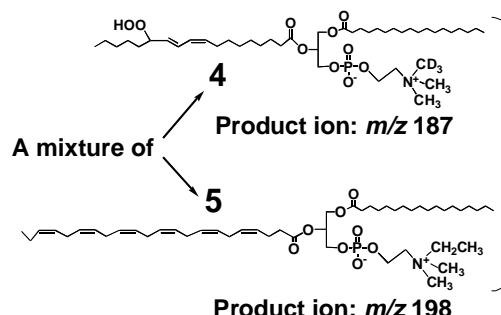

\section{Exposing Human Extraction of Tandem ESI MS}

Blood Phospholipid Measurement

or Skin
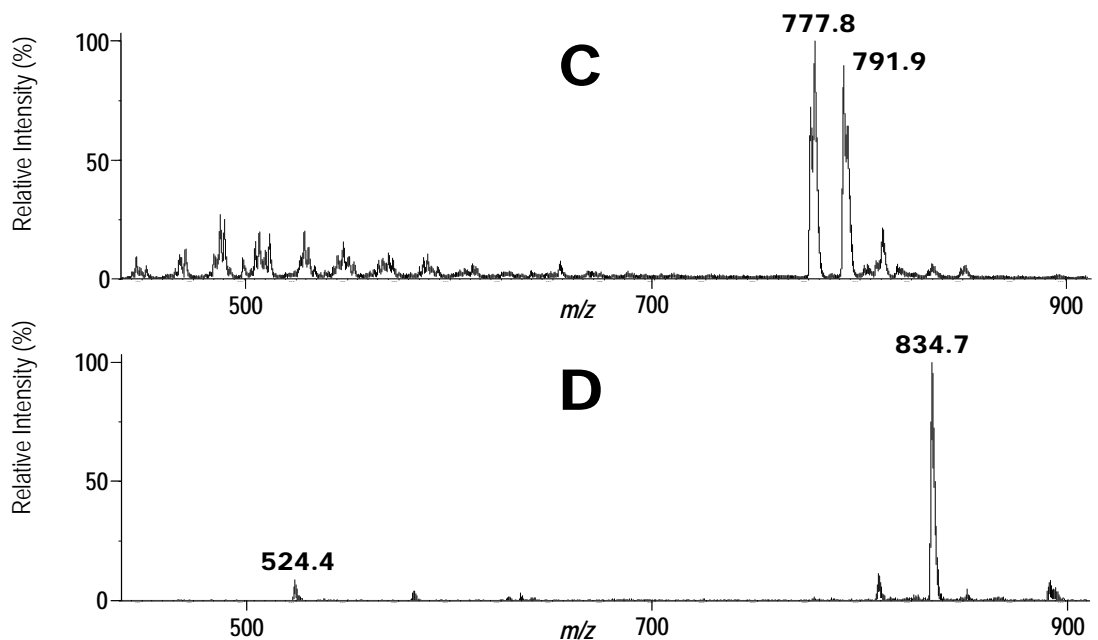

Figure 2. Upper scheme: A schematic description for use of a mixture of PCOOH/D3 3 and DHA-PC/Et 5 to trace simultaneously their structural changes in human blood (a 66 years old male volunteer). Precursor ion scan mode was operated at $m / z 187$ (C) or 198 (D) in tandem ESI MS for the phospholipid fraction extracted from the blood. Lower spectra: Tandem ESI MS spectra for the phospholipid fraction recovered from the human blood. C: A spectrum obtained at $\mathrm{m} / \mathrm{z} 187$. A signal at $\mathrm{m} / \mathrm{z} 793.8$ is of the unchanged intact phospholipid 3. D: Spectrum obtained at $\mathrm{m} / \mathrm{z} 198$. A signal at $\mathrm{m} / \mathrm{z} 834.7$ is of the unchanged intact phospholipid 3.
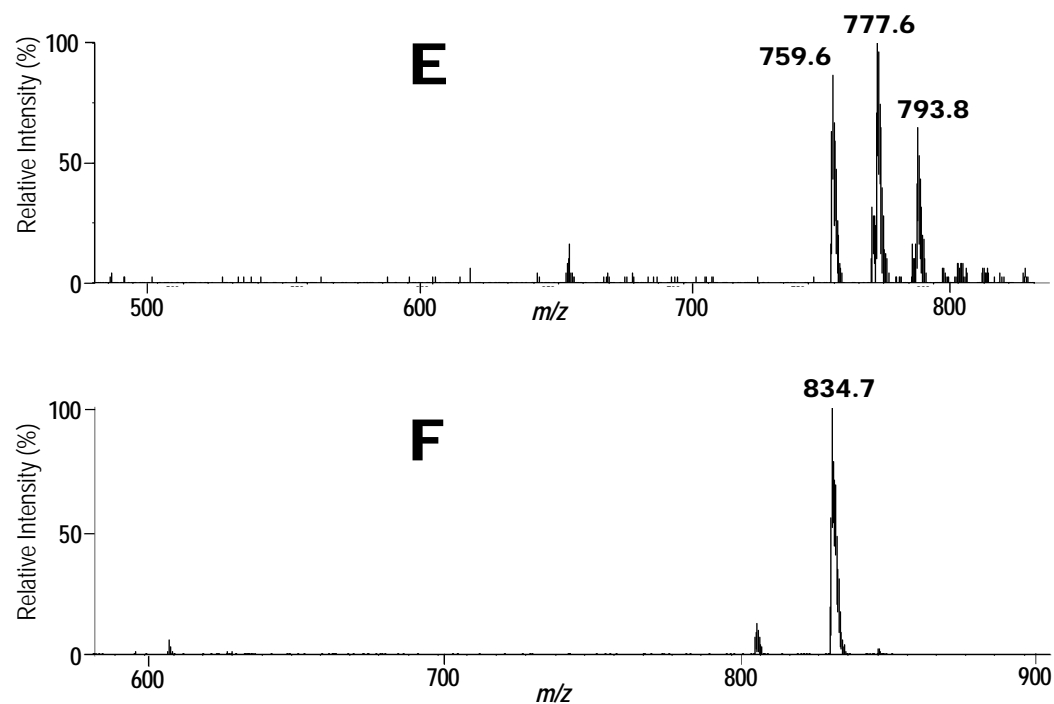

Figure 3. Tandem ESI MS spectra for the phospholipid fraction recovered from human skin surface. E. A spectrum obtained at $\mathrm{m} / \mathrm{z}$ 198. A signal at $\mathrm{m} / \mathrm{z} 793.8$ is of the unchanged intact phospholipid 3 . F. A spectrum obtained at $\mathrm{m} / \mathrm{z} 187$. A signal at $\mathrm{m} / \mathrm{z}$ 834.7 is of the unchanged intact phospholipid 5.

may be formed by dehydration of $\mathbf{6}$. Notably, product $\mathbf{1 1}$ was not formed when $\mathbf{3}$ was incubated in human blood (vide supra), showing a marked difference between the decomposition profile of $\mathrm{PC}-\mathrm{OOH} / \mathrm{D} 33$ on human arm skin surface and human blood. Thus, it seems almost impossible to accurately predict the profile of phosphol- 
ipid hydroperoxide degradation products in different tissues on the basis of mechanisms established in simple in vitro models. Also, even highly throughput technologies developed in recent years based on the combination of capillary electrophoresis or HPLC and highly sensitive mass spectrometry may require difficult task to do the same.

When we did precursor ion scan in ESI MS at $\mathrm{m} / \mathrm{z} 198$ for DHA-PC/Et 5, a major signal was observed at $\mathrm{m} / \mathrm{z}$ 834.7 that was a protonated form of 5 as shown in spectrum F (Figure 3). Other small signals could not be identified. Of particular interest is that, oxidatively unstable
DHA-PC/Et largely remained undecomposed against our expectation despite the oxidative stress with air and sunlight. This unexpected result strongly suggests that the skin surface might be endowed with some antioxidant system [26]. In fact, as Figure 4 shows, drastic decomposition of DHA-PC/Et 5 occurred on the surface of a glass plate with no antioxidant system. Possible structures for the degradation products formed from DHA-PC/ Et 5 on the glass plate were given in Scheme 4. The use of two different molecular probes at the same time should be useful for analysis of complex biological lipid dynamics. More important is that this strategy can be ex-

$$
\begin{aligned}
& \text { 12 } 13 \text { m/z674.4 }
\end{aligned}
$$

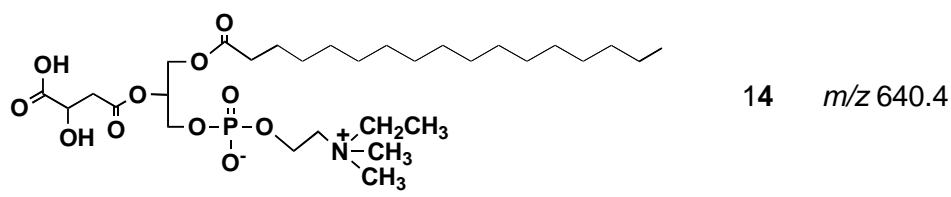

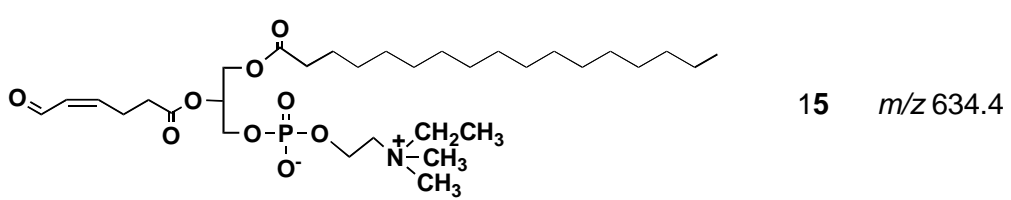

$$
\begin{aligned}
& \text { 总 } \\
& \text { O= }
\end{aligned}
$$

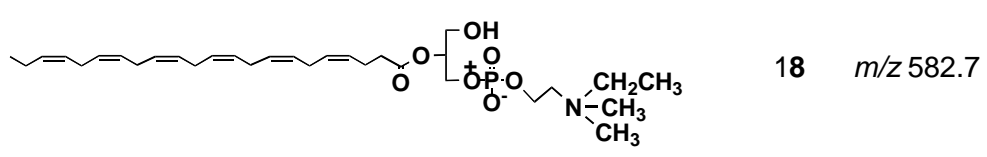

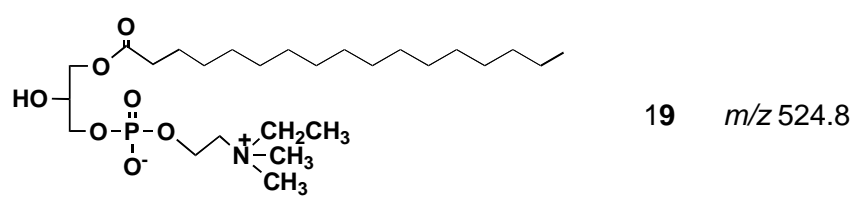

Scheme 4. Possible structure of degradation products formed from DHA-PC/Et 5 on the glass plate. All the $m / z$ values were calculated from their protonated form. 


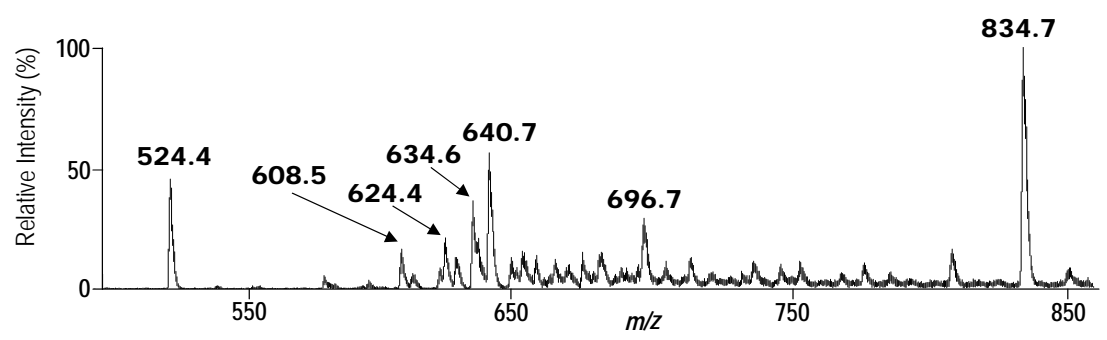

Figure 4. Tandem ESI MS Spectrum for the phospholipid fraction recovered from glass plate after exposing to the air at $20^{\circ} \mathrm{C}$ for $6 \mathrm{~h}$. A signal at $m / z$ 834.7 is a signal of the unchanged intact DHA-PC/Et 5.

panded to use of more than two different molecular probes at the same time under strictly the same biological conditions.

\section{Mass Spectrometry Assisted Tracing of Structural Changes in Phospholipid Molecular Probe Bearing Docosahexaenoyl Group Applied on Fish Skin Surface}

Fishes, salt water fishes in particular, are rich in polyunsaturated fatty acids (PUFA, mainly DHA and eicosapentaenoic acid (EPA)), and their worldwide consumption has increasingly expanded year by year because of their beneficial effects in human health. Drying fish under sunlight for their preservation and flavor generation is a traditional practice in many countries in the world. In this process, oxidatively vulnerable PUFA should naturally decompose to afford many degradation products including toxic aldehydic products with highly unpleasant odors. Against our expectation, dried fishes usually have appetizing flavor especially when they are baked. This apparent contradiction has long remained unexplained. Our synthetic phospholipid molecular probe 5 having docosahexaenoyl group has been used to investigate this problem [27]. The molecular probe was applied on the surface of herring fillets and dried over one week under the sunlight. The lipid fraction was extracted from the surface of the dried fish and the extract was directly subjected to electrospray ionization mass spectrometry in precursor ion scan mode at $491 \mathrm{~m} / \mathrm{z}$ as a product ion. The spectrum is shown in Figure 5. A signal at this $\mathrm{m} / \mathrm{z} 582.7$ was assigned as a hydrolytic fragment 18. Also, a signal at $\mathrm{m} / \mathrm{z} 524.8$ was assigned as another hydrolytic fragment, lyso-PC 19. In addition to these signals, a number of small signals were observed, and we tried to assign the structures based on $m / z$ and oxidative fragmentation mechanism of DHA. The deduced structures from the spectrum were given as compounds 20-23 (Scheme 5). It is well known that processed fishes have amino compounds such as those shown in Scheme 6. It is also known that the oxidation of polyunsaturated fatty acids like DHA affords various aldehydes, most of which have unpleasant odor. On the basis of chemistry, amines

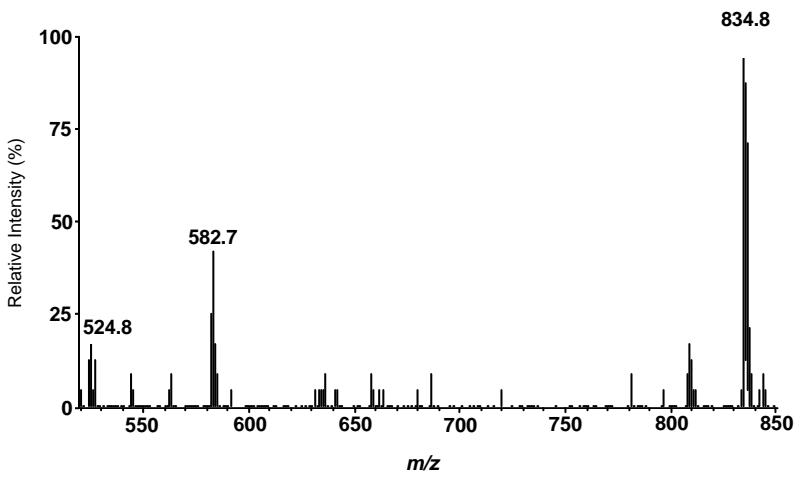

Figure 5. Tandem ESI MS spectrum for the phospholipid fraction recovered from the processed fish skin surface.

and aldehydes react easily to form imines (Scheme 6). Generally, the odor of most of imines may be different from that of aldehydes and rather similar to amines. Therefore, we speculated that the aldehyde species formed during the drying process were converted to imines. If we assume that all the aldehydes were consumed to react with excess amines, the imine formation appears to explain why the dried fish had no unpleasant aldehyde odor. Indeed, most dried fishes and squid or "surimi" in Japanese have amine like odor. Thus, lipid oxidation and imine formation may be responsible for the generation of the characteristic taste and flavor of fish fillet during the drying process. Since our method is speedy with no isolation procedure, it is potentially useful for analyses of changes in food characteristics and other complex biochemical phenomena.

\section{Mass Spectrometry Assisted Analysis of Phospholipase $\mathbf{A}_{2}$ Enzymatic Activity}

Phospholipase $\mathrm{A}_{2}$, a typical phospholipid-related enzyme, plays a role in lipid digestion as a component of the pancreatic juice. It is also involved in biological signaling by participating in the onset of the arachidonic acid cascade. As a component of snake venom, it contributes to hemolysis of blood. There are many studies on the inhibition of this enzyme and the catalytic activity analyses have been exploited. In biological systems, lipid composition is extremely complex, and it is difficult to trace the activ- 
<smiles>CCCCCCCCCCCCCCC(=O)OCC(COP(=O)(O)OCC[N+](C)(C)C)OCC(=O)OCCC=CCN</smiles><smiles>CCCCCCCCCCCCCCC(=O)OCC(COP(=O)(O)OCC[N+](C)(C)C)OC(=O)CCC=CC=CC=N</smiles><smiles>CCCCCCCCCCCCCCCC(=O)OCC(COP(=O)(O)OCC[N+](C)(C)C)OC(=O)CCC=CCC=CC=CC=CCC(CCO)NCCC</smiles><smiles>CC[N+](C)(C)CCOP(=O)(O)OCC(CO)(CO)OC(=O)CC/C=C/C/C=C/C=C/C=C/C=C/CCCN</smiles>

Scheme 5. Possible structures for the degradation products recovered from the processed fish surface all the $m / z$ values were calculated from their protonated form.
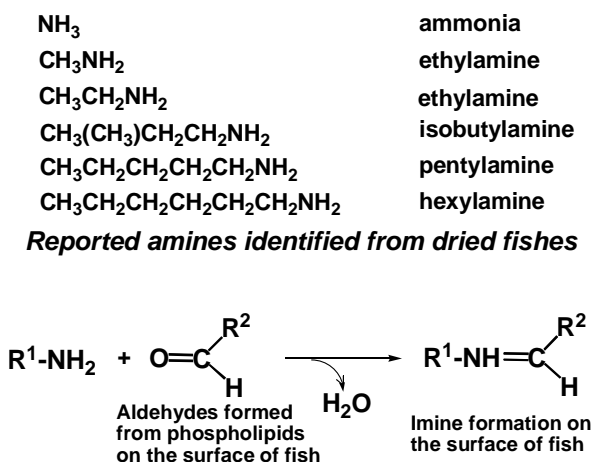

Scheme 6. Identified amines isolated from processed fish and the general reaction between amines and aldehydes affording imines.

ity toward a specific phospholipid molecule in the presence of many similar species. We used a phospholipid molecular probe 24 (Figure 6) for first rough screening of phospholipase $\mathrm{A}_{2}$ activity in plant species. The molecular probe was added to a mixture of pancreatic phospholipase $\mathrm{A}_{2}$ and a given plant extract. After stirring for sometime, the lipid fraction was extracted with a mixture of methanol:chloroform (1:2). The extract was directly subjected to mass spectrometry and precursor ion Figure 7 was obtained. Here, a signal of the product, scan mode was conducted at $\mathrm{m} / \mathrm{z} 187$. When Juniperus rigida leaf was used as a candidate of inhibitory activity against the phospholipase $\mathrm{A}_{2}$, a spectrum as shown in lyso-PC is observed at $\mathrm{m} / \mathrm{z}$ 523.3. Like this way, several plant leaves were examined and the results were given in Table 1. Thus, a combination of phospholipid molecular

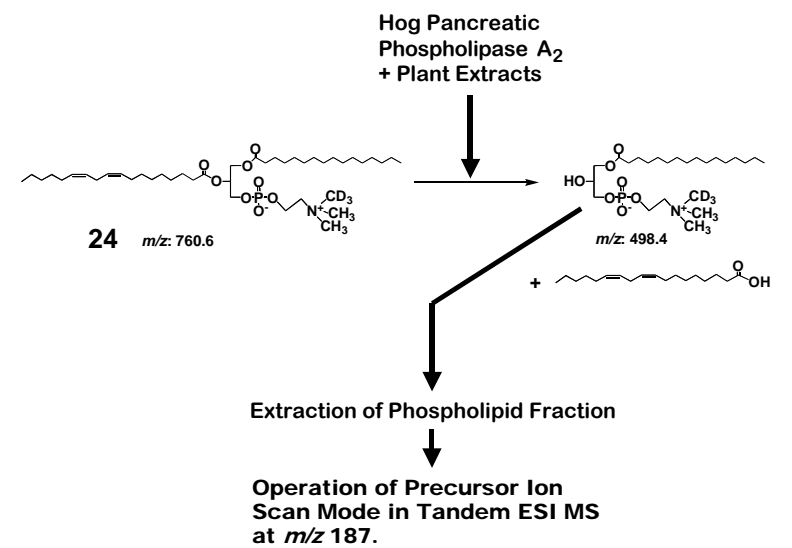

Figure 6. Hog pancreatic phospholipase $A_{2}$-catalyzed hydrolysis of linoleoyl PC 23 in the presence of plant extracts, and analysis of the reaction mixture in precursor ion scan mode with ESI mass spectrometry.

probe and tandem mass spectrometry was demonstrated to be a simple and easy method for first screening of phospholipase $\mathrm{A}_{2}$ inhibitory activity.

\section{Conclusion}

A combination of phospholipid molecular probes using collision-induced tandem electrospray ionization mass spectrometry demonstrated that the fate of unstable phospholipid hydroperoxide such as $\mathbf{3}$, could be traced in biological systems such as human blood and human skin surface even in the presence of complex mixture of biological lipids. A molecular probe $\mathbf{5}$ having docosahexaenoic acid was also found to be useful to trace the 
Table 1. Primary screening for inhibitory activity of some plants on phospholipase $A_{2}$ using molecular probe 23 in conbination mode at $\mathrm{m} / \mathrm{z} 187$ with tandem ESI mass spectrometry in precursor ion scan.

\begin{tabular}{cc}
\hline Plant & $\begin{array}{r}\text { Inhibition of plant on the activity } \\
\text { of phospholipase } \mathrm{A}_{2}\end{array}$ \\
\hline Camellia sinensis leaf & strong inhibition \\
Coffea arabica bean & strong inhibition \\
Rosmarinus officinalisleaf & slight enhancement \\
Pinus densiflora leaf & P slight inhibition \\
Juniperus rigida leaf & moderate inhibition \\
Photonia glabra leaf & slight inhibition \\
Photonia glabra fruit & strong inhibition \\
Phytolacca americana fruit & slight inhition \\
\hline
\end{tabular}

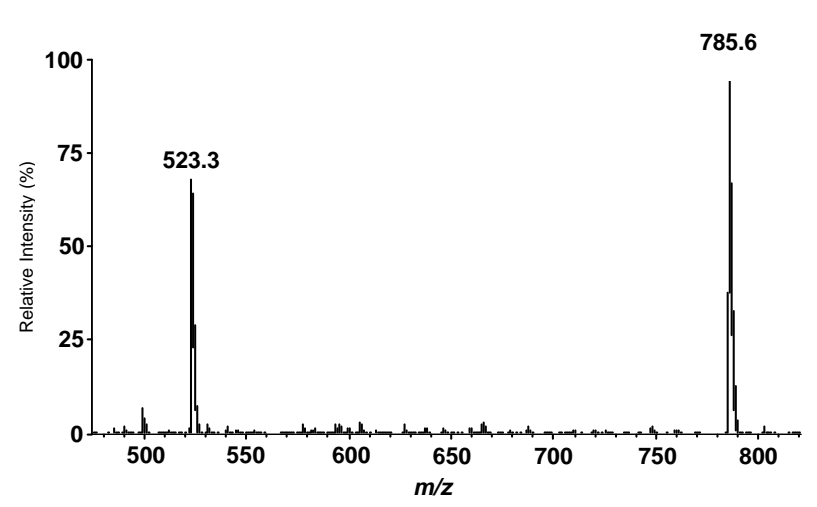

Figure 7. ESI mass spectrum of the reaction mixture obtained from phospholipase $A_{2}$-catalyzed hydrolysis of linoleoyl PC 23 in the presence of the plant extracts.

chemical changes at its oxidatively unstable all cis nonconjugated polyunsaturated olefinic structure in the biological systems and the processed fish food. This strategy was also proved to be useful for very simple analysis of phospholipase $A_{2}$ activity. The present method is strictly specific even in the presence of a large excess of a complex biological substances and it is speedy within less than $1 \mathrm{~h}$ after extraction from biological samples with no product-isolation procedure. Therefore, the present method may potentially be useful for tracing time-dependent phenomena in dynamic phospholipid biochemistry, and can be widely applied to any biological and food systems.

\section{Acknowledgements}

This study was partly supported by the Japan Society for the Promotion of Science (JSPS) Asian CORE Program entitled "Cooperative Research and Educational Center for Important Plant Genetic Resources in East Asia". We are grateful to staff at the laboratory of SC-NMR and the laboratory of the API III Mass spectrometry in Okayama University.

\section{REFERENCES}

[1] A. Shevchenko and K. Simons, "Lipidomics: Coming to Grips with Lipid Diversity," Nature Reviews Molecular Cell Biology, Vol. 11, No. 8, 2010, pp. 593-598.

[2] E. Fahy, D. Cotter, M. Sud and S. Subramaniam, "Lipid Classification, Structures and Tools," Biochimica et Biophysica Acta (BBA)-Molecular and Cell Biology of Lipids, Vol. 1811, No. 11, 2011, pp. 637-647.

http://dx.doi.org/10.1016/j.bbalip.2011.06.009

[3] A. Lamaziere, D. Richard, U. Barbe, K. Kefi, P. Bausero, C. Wolf and F. Visiol, "Differential Distribution of DHA-Phospholipids in Rat Brain after Feeding: A Lipidomic Approach," Prostaglandins Leukot Essent Fatty Acid, Vol. 84, No. 1, 2011, pp. 7-11. http://dx.doi.org/10.1016/j.plefa.2010.11.001

[4] P. Fagone and S. Jackowski, "Membrane Phospholipid Synthesis and Endoplasmic Reticulum Function" The Journal of Lipid Research, Vol. 50, 2009, pp. S311-S316. http://dx.doi.org/10.1194/jlr.R800049-JLR200

[5] K. Halder, A. F. de Amorim and A. M. Cross, "Transport of Fluorescent Phospholipid Analogues from the Erythrocyte Membrane to the Parasite in Plasmodium falciparum-Infected Cells," The Journal of Cell Biology, Vol. 108, No. 6, 1989, pp. 2183-2102. http://dx.doi.org/10.1083/jcb.108.6.2183

[6] D. Marsh, "Electron Spin Resonance in Membrane Research: Protein-Lipid Interactions from Challenging Beginnings to State of The Art," European Biophysics Journal, Vol. 39, No. 4, 2010, pp. 513-525. http://dx.doi.org/10.1007/s00249-009-0512-3

[7] M. Pulfer and R. C. Murphym, "Electrospray Mass Spectrometry of Phospholipids," Mass Spectrometry Reviews, Vol. 22, No. 5, 2003, pp. 332-364. http://dx.doi.org/10.1002/mas.10061

[8] V. E. Kagan, G. G. Borisenko, Y. Y. Tyurina, T. A. Tyurin, J. Jiang, A. I. Potapovich, V. Kini, A. A. Amoscato and Y. Fuji, "Oxidative Lipidomics of Apoptosis: Redox Catalytic Interactions of Cytochrome $\mathrm{c}$ with Cardiolipin and Phosphatidylserine," Free Radical Biology \& Medicine, Vol. 37, No. 12, 2004, pp.1963-1985.

http://dx.doi.org/10.1016/j.freeradbiomed.2004.08.016

[9] Katja Dettmer, P. A. Aronov and B. D. Hammock, "Mass Spectrometry-Based Metabolomics," Mass Spectrometry Reviews, Vol. 26, No. 1, 2007, pp. 51-78. http://dx.doi.org/10.1002/mas.20108

[10] M. Rosario, M. Domingues, A. Reis and P. Domingues, "Mass Spectrometry Analysis of Oxidized Phospholipids," Chemistry and Physics of Lipids, Vol. 156, No. 1-2, 2008, pp. 1-12. http://dx.doi.org/10.1016/j.chemphyslip.2008.07.003

[11] A. D. Postle and A. N. Hunt, "Dynamic Lipidomics with Stable Isotope Labeling," Journal of Chromatography B, Vol. 877, No. 26, 2009, pp. 2716-2721. http://dx.doi.org/10.1016/j.jchromb.2009.03.046 
[12] R. J. Mishur and S. L. Rea, "Applications of Mass Spectrometry to Metabolomics and Metabonomics: Detection of Biomarkers of Againg and of Age-Related Diseases," Mass Spectrometry Reviews, Vol. 31, No. 1, 2012, pp. 7095. http://dx.doi.org/10.1002/mas. 20338

[13] A. Thomas, J. Déglon, S. Lenglet, F. Mach, P. Mangin, J. Wolfender, S. Steffens and C. Staub, "High-Throughput Phospholipidic Fingerprinting by Online Desorption of Dried Spots And Quadrupole-Linear Ion Trap Mass Spectrometry: Evaluation of Atherosclerosis Biomarkers In Mouse Plasma," Analytical Chemistry, Vol. 82, No. 15, 2010, pp. 6687-6694. http://dx.doi.org/10.1021/ac101421b

[14] V. N. Bochkov and N. Leitinger, "Anti-Inflammatory Properties of Lipid Oxidation Products," Journal of Molecular Medicine, Vol. 81, No. 10, 2003, pp. 613-626. http://dx.doi.org/10.1007/s00109-003-0467-2

[15] G. K. Marathe, A. Harrison, R. C. Murphy, S. M. Prescott, G. A. Zimmerman and T. M. McIntyre, "Bioactive Phospholipid Oxidation Products," Free Radical Biology \& Medicine, Vol. 28, No. 12, 2006, pp. 1762-1770. http://dx.doi.org/10.1016/S0891-5849(00)00234-3

[16] C. Erridge, S. Kennedy, C. M. Spickett and D. J. Webb, "Oxidized Phospholipid Inhibition of Toll-Like Receptor (TLR) Signaling Is Restricted to TLR2 and TLR4, "The Journal of Biological Chemistry," Vol. 283, 2008, pp. 24748-24759.

http://dx.doi.org/10.1074/jbc.M800352200

[17] R. A. Siddiqui, K. Harvey and W. Stillwell, “Anticancer Properties of Oxidation Products of Docosahexaenoic Acid," Chemistry and Physics of Lipids, Vol. 153, No. 1, 2008, pp. 47-56.

http://dx.doi.org/10.1016/j.chemphyslip.2008.02.009

[18] G. K. Marathe, K. A. Harrison, R. C. Murphy, S. M. Prescott, G. A. Zimmerman and T. M. ScIntyre, "Bioactive Phospholipid Oxidation Products", Free Radical Biology \& Medicine, Vol. 28, No. 12, 2000, pp. 1762-1770. http://dx.doi.org/10.1016/S0891-5849(00)00234-3

[19] T. Miyazawa, T. Suzuki, K. Fujimoto and M. Kinoshita, "Age-Related Change of Phosphatidylcholine Hydroperoxide and Phosphatidylethanolamine Hydroperoxide Levels in Normal Human Red Blood Cells," Mechanisms of Ageing and Development, Vol. 86, No. 3, 1996, pp. 145150. http://dx.doi.org/10.1016/0047-6374(95)01687-2

[20] V. Frisardi, F. Panza, D. Seripa, T. Farooqui and A. A. Farooqui, "Glycerophospholipids and Glycerophospholipid-Derived Lipid Mediators: A Complex Meshwork in
Alzheimer's Disease Pathology," Progress in Lipid Research, Vol. 50, No. 4, 2011, pp. 313-330. http://dx.doi.org/10.1016/j.plipres.2011.06.001

[21] R. Shimizu, A. Nagai, H. Tominaga, H. Tominaga, M. Imura, A. N. Onyango, M. Izumi, S. Nakajima, S. Tahara, T. Kaneko and N. Baba, "A Combination of Molecular Probe-Tandem Electrospray Ionization Mass Spectrometry: A Technique for Tracing Structural Changes in Phospholipid Hydroperoxides," Bioscience, Biotechnology, and Biochemistry, Vol. 73, No. 3, 2009, pp. 781-784. http://dx.doi.org/10.1271/bbb.80672

[22] N. Baba, H. Daido, T. Kosugi, M. Miyake and S. Nakajima, "Analysis of Glycerophospholipid Hydroperoxides by Ion Spray Mass Spectrometry," Bioscience, Biotechnology, and Biochemistry, Vol. 62, No. 1, 1998, pp. 160163. http://dx.doi.org/10.1271/bbb.62.160

[23] A. N. Onyango, N. Kumura, H.Tominaga and N. Baba, "Dihydroperoxidation Facilitates the Conversion of Lipids to Aldehydic Products via Alkoxyl Radicals," Food Research International, Vol. 43, No. 3, 2010, pp. 925-929. http://dx.doi.org/10.1016/j.foodres.2009.12.011

[24] H. Kern, T. Volk, S. Knaueru-Schiefer, T. Mieth, B. Bustow, W. J. Kox and M. Schlame, "Stimulation of Monocytes and Platelets by Short-Chain Phosphatidylcholines with and without Terminal Carboxyl Group," Biochimica et Biophysica Acta (BBA)-Molecular and Cell Biology of Lipids, Vol. 1394, No. 1, 1998, pp. 33-42. http://dx.doi.org/10.1016/S0167-4889(98)00093-7

[25] S. Hong and Y. Lu, "Omega-3 Fatty Acid-Derived Resolvins and Protectins in Inflammation Resolution and Leukocyte Functions: Targeting Novel Lipid Mediator Pathways in Mitigation of Acute Kidney Injury," Frontiers in Immunology, Vol. 4, 2013, p. 13.

[26] C. D. Luca and G. Valacchi, "Surface Lipids as Multifunctional Mediators of Skin Responses to Environmental Stimuli," Mediators of Inflammation, Vol. 2010, 2010, pp. 1-11. http://dx.doi.org/10.1155/2010/321494

[27] A. K. M. Azad Shah, T. Ishihara, M. Ogasawara, H. Kurihara, N. Baba and K. Takahashi, "Mechanism Involved in the Formation of Characteristic Taste and Flavor During the Production of Dried Herring (Clupea pallasii) Fillet," Food Science and Technology Research, Vol. 16, No. 3, 2010, pp. 201-208.

http://dx.doi.org/10.3136/fstr.16.201 